\title{
Comparison of non-invasive NIR and Raman spectrometries for determination of alcohol content of spirits
}

Alison Nordon,* Angela Mills, Ross T. Burn, Fiona M. Cusick and David Littlejohn, Department of Pure and Applied Chemistry/CPACT, University of Strathclyde, 295 Cathedral Street, Glasgow, G1 1XL, UK.

* Author to whom correspondence should be sent (alison.nordon@strath.ac.uk)

\section{Abstract}

The suitability of non-invasive NIR and Raman spectrometries for determination of \% ethanol content has been investigated. Samples of whisky, vodka and sugary alcoholic drinks were analysed in $200 \mathrm{~mL}$ (flat) and $700 \mathrm{~mL}$ (round) glass bottles. The NIR spectrometer used double transmission measurements and was limited mainly to analysis of the signal produced at about $10000 \mathrm{~cm}^{-1}$ by water and ethanol in the samples. The Raman measurements, produced using a $785 \mathrm{~nm}$ laser, were based on a sharp signal from ethanol at $880 \mathrm{~cm}^{-1}$. A multivariate calibration model, based on a combined PCA-PLS algorithm, was required for analysis by NIR spectrometry, whereas a univariate calibration model was suitable for Raman spectrometry. Both techniques were limited to measurements in clear glass bottles as strong absorption/fluorescence occurred with coloured glass bottles. Bottle-to-bottle variations contributed the largest uncertainty to the measurements obtained for a $20 \%$ V/V ethanol solution in flat bottles: $2.3 \%$ RSD for NIR spectrometry and $2.2 \%$ RSD

for Raman spectrometry. For $700 \mathrm{~mL}$ round bottles, which have stricter 
manufacturing tolerances on glass thickness, the bottle-to-bottle variability for Raman spectrometry was $1.4 \%$ RSD. When spirit samples with ethanol concentrations in the range 19.9-61.7\% V/V were analysed, the precision (average RSD) was 0.4 and $0.5 \%$ for NIR (flat bottles) and Raman (round bottles) spectrometries, respectively, and the average accuracy was 2.1 and $2.9 \%$, respectively. When a calibration model constructed from NIR data acquired on one day was applied to data sets collected over a 15 day period, the average error was $3.9 \%$.

Keywords: alcoholic beverages, non-invasive analysis, Raman spectrometry, NIR spectrometry, derivative spectrometry, ethanol.

\section{Introduction}

In the UK, Customs and Excise require the alcohol content of beverages to be determined with an accuracy of $\pm 0.05 \% \mathrm{~V} / \mathrm{V}$. Most of the methods that are employed currently are based on density measurements. Although such methods are accurate, they possess a number of disadvantages. Usually, density measurements are performed off-line in a centralised analytical laboratory, and hence, there can be a considerable delay before the alcohol content of a sample is reported. For those alcoholic beverages that possess a high dissolved solids content (e.g. sugars), the sample must be distilled to remove the alcohol prior to performing a density measurement. Off-line density measurements are also destructive in the sense that once a bottle has been opened or a sample has been removed from the process line, it cannot be resealed or returned, respectively. 
Spectroscopic techniques offer a faster and simpler method for the determination of alcohol content. Off-line mid infrared (MIR) spectrometry has been used to determine the ethanol content of spirits and a range of beer samples in a micro flow transmission cell [1,2]. Off-line near infrared (NIR) spectrometry has been used to determine the alcohol content of beers [3,4], wines, whiskies, gin, rum and liqueurs [5]. The ethanol content of different types of alcoholic beverages has been determined using off-line NIR measurements, which employed a transflectance immersion probe [6] and a quartz cell [7]. In the latter study [7], it was demonstrated that accurate prediction of alcohol content could be obtained between 15 and $35^{\circ} \mathrm{C}$ by removal of temperature induced spectral changes using orthogonal signal correction [8] or piecewise direct standardisation [9]. Alcoholic beverages have also been analysed using off-line Raman spectrometry with univariate [10,11] or multivariate [6] calibration models. A stopped-flow NIR system was used for the analysis of beers to provide a greater throughput of samples [12]. For both MIR and NIR spectrometry, the presence of sugars interferes with the ethanol signal. In cases where a separate sugar signal can be isolated, this can be used to correct for the sugar contribution to the ethanol signal $[1,2,4,5,12]$. Alternatively, different calibration models may be constructed for different types of alcoholic beverages. Partial least squares (PLS) calibration models have been prepared from off-line MIR spectra of wines to determine the concentration of glucose, fructose, glycerol, ethanol and organic acids $[13,14]$. Recently, a small portable hand-held UV-visible spectrometer was developed for authentication of whisky samples in the field [15]. One of the major advantages of MIR, NIR, UV-visible and Raman spectrometries is that no sample preparation, other than degassing for certain types of samples (e.g. beers [3]), is required. However, use 
of these techniques either off-line or at-line still requires the removal of a sample from the process line.

On-line determination of alcohol content could involve either insertion of a probe into the process stream (i.e. in-line analysis), analysis through a vessel or pipe wall (i.e. non-invasive analysis) or analysis of a sample in its container on a bottling line. In-line NIR spectrometry has been used to determine the concentration of ethanol in wine samples [16] and alcoholic beverages [17]. In chemical process analysis, ethanol has been determined by in-line Raman spectrometry [18] and non-invasive NIR spectrometry [19]. The non-invasive approach is only possible with optical techniques if the vessel is made of a transparent material or has a suitable window. The concentrations of ethanol, propylene glycol and water in a pharmaceutical oral liquid were determined non-invasively using NIR spectrometry [20] by direct analysis of the sample in amber plastic containers that were placed into the instrument sample compartment.

In this work, NIR and Raman spectrometries have been used non-invasively to analyse different types of spirits contained within glass bottles. The study was conducted to evaluate the techniques for possible use in non-invasive in situ quality assurance in bottling plants. However, prior to conducting any measurements with moving bottles on a bottling line, an investigation involving static bottles was carried out and is reported here. Each technique was assessed in terms of the accuracy and precision with which the alcohol content could be determined and the ease of constructing and maintaining calibration models, particularly when only a small number of samples are available. The influence of the glass bottle on the measurement has also been considered. 


\section{Experimental}

\subsection{Samples}

Thirty-two samples were provided by Guinness United Distillers and Vintners (Menstrie, UK). The samples were of three different types; whiskies, vodkas and alcoholic sugary drinks. The alcohol (ethanol) concentrations for the whiskies, vodkas and sugary drinks were in the ranges $20.2-61.7,19.9-63.3$ and $11.4-35.6 \% \mathrm{~V} / \mathrm{V}$, respectively. The alcohol concentrations, supplied with the samples, were obtained with at-line NIR spectrometry using either of two calibration models that comprised concentration and spectral data for 45 and 69 alcoholic samples of different types, for prediction of whisky and all other types of drinks, respectively.

All samples were supplied in $200 \mathrm{~mL}$ clear glass flat bottles, which had a length and width of approximately 70 and $35 \mathrm{~mm}$. Additional $200 \mathrm{~mL}$ clear glass flat bottles and also $700 \mathrm{~mL}$ clear and green glass round bottles (diameter of $70 \mathrm{~mm}$ ) were supplied. These bottles were used with a solution of 20 or $28.8 \% \mathrm{~V} / \mathrm{V}$ ethanol in distilled water to assess the effects of the diameter and colour of the bottle on the signals obtained, and to investigate the variability of different types of bottles. A solution of $20 \% \mathrm{~V} / \mathrm{V}$ ethanol was also used to assess the contributions of different factors to the overall analytical variance of the measurements. Samples of whisky, vodka and sugary drinks were analysed as received in the 200 and $700 \mathrm{~mL}$ clear glass bottles. For flat bottles, there were two possible measurement orientations; the NIR source or Raman laser could point towards the narrow $(35 \mathrm{~mm})$ side of the bottle (i.e. measurement across the widest part of the bottle) or towards the widest $(70 \mathrm{~mm})$ side of the bottle (i.e. measurement across the narrowest part of the bottle). 


\subsection{NIR spectrometry}

NIR spectra were acquired with a resolution of $6 \mathrm{~nm}$ in the 939-1708 nm $\left(10650-5855 \mathrm{~cm}^{-1}\right)$ region using a Zeiss Corona 45 NIR reflectance spectrometer (Clairet Scientific, Northampton, UK) equipped with an InGaAs diode array detector. Two spectrometers of this type (denoted spectrometer 1 and spectrometer 2), which were of identical specification, were used during the course of this work. Spectra were acquired using Aspect Plus version 1.71 software (Zeiss, Germany), which were then exported into Excel (Microsoft Corporation, USA) as tab-delimited text files. NIR spectra were acquired for 29 samples (samples 1-23 and 27-32; 24-26 were analysed only by Raman). Samples 1-23 were analysed on the same day using spectrometer 1 . Samples 27-32 were analysed on 11 different days over a 15 day period using spectrometer 2. All measurements of the whisky, vodka and sugary drinks were conducted in the $200 \mathrm{~mL}$ clear glass flat bottles, and across the narrowest (width of $35 \mathrm{~mm}$ ) part of the bottle. The bottles were placed upright in front of the lens and a white tile was positioned behind the bottle to reflect the light back to the collection fibres that are situated around the inner edge of the lens. An integration time of $30 \mathrm{~ms}$ (spectrometer 1) or $48 \mathrm{~ms}$ (spectrometer 2) was employed, which was set using a white tile placed directly in front of the lens. A dark current measurement, which was subtracted from all intensities, was made with a cover over the lens. An empty bottle was used to obtain the reference intensities over the wavelength range (i.e. $I_{0}$ ) and this was used with the sample intensities (I) to calculate the absorbance of the samples. A total of 10 scans were accumulated for each measurement and five repeat measurements were made for each sample. The time for each replicate measurement was 300 or $480 \mathrm{~ms}$. No temperature control of the samples was attempted. However, 
the sample was placed in front of the lens just before the measurement period to minimise the heating of the sample by the NIR source.

Solutions of 20 and $28.8 \% \mathrm{~V} / \mathrm{V}$ ethanol were also analysed in: $200 \mathrm{~mL}$ clear glass flat bottles across the narrowest (width of $35 \mathrm{~mm}$ ) part of the bottle; a $700 \mathrm{~mL}$ clear glass round bottle across the widest (diameter of $70 \mathrm{~mm}$ ) and narrowest (i.e. at the neck; diameter of $40 \mathrm{~mm}$ ) parts of the bottle; a $700 \mathrm{~mL}$ green glass round bottle across the narrowest part of the bottle. With the $700 \mathrm{~mL}$ round bottles, the reference measurement was made by placing the white tile directly in front of the lens.

\subsection{Raman spectrometry}

Raman spectra were acquired using a Kaiser HoloProbe spectrometer (Clairet Scientific) with a laser excitation wavelength of $785 \mathrm{~nm}$. Samples were analysed using a non-contact probe head (focal length of $6.5 \mathrm{~cm}$ ), which was connected to the Raman spectrometer using a $5 \mathrm{~m}$ length of silica optical fibre. Spectra were acquired using HoloGRAMS software (Kaiser Optical Systems, Ann Arbor, Michigan, USA), which were exported into GRAMS 32 software (Galactic Industries Corporation, USA). Spectra were then exported into Excel as .prn files. The optimum distance of the sample from the probe head was determined using a bottle containing toluene; the optimum position was achieved when the maximum toluene $\left(1004 \mathrm{~cm}^{-1}\right)$ to glass $\left(1370 \mathrm{~cm}^{-1}\right)$ intensity ratio was obtained. Raman spectra were acquired on the same day for 20 samples (samples 1-4, 8-14, 16, 18 and 20-26). All measurements of the whisky, vodka and sugary drinks (except samples 12 and 13) were conducted in the $700 \mathrm{~mL}$ clear glass round bottles, and across the widest (diameter of $70 \mathrm{~mm}$ ) part of the bottle. Raman spectra were acquired using dark current subtraction, 4 
accumulations and an exposure time of $40 \mathrm{~s}$. However, for those samples that fluoresced (samples 12 and 13), the samples were analysed in $200 \mathrm{~mL}$ clear glass flat bottles across the widest part of the bottle and an exposure time of $10 \mathrm{~s}$ was employed. Three repeat measurements were made for each sample and the time for one replicate measurement (with an exposure time of $40 \mathrm{~s}$ ) was 2.7 mins. Information from the manufacturer indicates that with the latest version of the Raman spectrometer, the measurement time could be reduced by at least a factor of 3 .

Solutions of 20 and $28.8 \% \mathrm{~V} / \mathrm{V}$ ethanol were analysed in: $200 \mathrm{~mL}$ clear glass flat bottles across the narrowest (width of $35 \mathrm{~mm}$ ) and widest (width of $70 \mathrm{~mm}$ ) parts of the bottle; $700 \mathrm{~mL}$ clear glass round bottles across the widest (diameter of $70 \mathrm{~mm}$ ) part of the bottle; a $700 \mathrm{~mL}$ green glass round bottle across the widest (diameter of $70 \mathrm{~mm}$ ) part of the bottle. Five repeat measurements were made for the solutions of 20 or $28.8 \% \mathrm{~V} / \mathrm{V}$ ethanol. An exposure time of $55 \mathrm{~s}$ was employed for analysis of the ethanol solutions except for the solution contained in a green glass bottle; in this case, an exposure time of $1 \mathrm{~s}$ was used owing to the intense fluorescence from the bottle.

\subsection{Data analysis}

All data were imported into Matlab version 6.5 (Mathworks Inc., Natick, Massachusetts, USA) for analysis using the PLS_Toolbox version 2.1.1 (Eigenvector Research, USA). NIR and Raman data were processed using a Savitsky-Golay first derivative filter, which employed a width of 5 and 25 data points, respectively, and a second order polynomial. 
The slopes \pm standard error for graphs of alcohol content against Raman signal intensity were determined using Excel and statistical tests of the data was conducted using the Data Analysis Toolpak in Excel.

\subsubsection{Calculation of variance}

To assess the contribution of instrumental measurement, bottle position and bottle-to-bottle variation to the overall analytical variance, repeat measurements were conducted on a solution of $20 \% \mathrm{~V} / \mathrm{V}$ ethanol contained in 5 different bottles of the same type. The contributions of the different factors were calculated from the variance of the signal in the first derivative NIR and Raman spectra at 9796 and $873 \mathrm{~cm}^{-1}$, respectively.

The short-term instrumental measurement variance was obtained from repeat measurements of the $20 \% \mathrm{~V} / \mathrm{V}$ ethanol solution contained in one bottle, without moving the bottle in between repeat measurements. Five and 25 repeat measurements were made for this purpose for NIR (spectrometers 1 and 2) and Raman spectrometries, respectively. Next, 25 repeat measurements were made of the $20 \%$ $\mathrm{V} / \mathrm{V}$ ethanol solution contained in one bottle, but after every 5 measurements the bottle was repositioned in front of the lens. In this case, the variance of the signal was from both instrumental measurement and bottle position variation. Five repeat measurements were also made of the $20 \% \mathrm{~V} / \mathrm{V}$ ethanol solution contained in 5 different bottles (total of 25 measurements). The variance of the signal, in this case, can be attributed to short-term instrumental measurement, bottle position and bottleto-bottle variation. In addition, the long-term NIR instrumental measurement variance was assessed using spectrometer 2 by carrying out 5 repeat measurements of the $20 \%$ 
$\mathrm{V} / \mathrm{V}$ ethanol solution contained in one bottle, without moving the bottle in between repeat measurements, on 11 different days over a 15 day period. The variance of the signal can be attributed to the instrument variation (both short- and long-term) and any variation in the positioning of the bottle in front of the lens over the 15 days. As the total variance is equal to the sum of the individual contributions, comparison of the different sets of data allowed calculation of the variance associated with the various factors. The standard deviation was calculated from the variance. The average signal intensities for the replicate measurements were also calculated, allowing the contribution of the different sources of variance to the overall analytical measurement variability to be expressed as a relative standard deviation (RSD).

\subsubsection{Construction of calibration models}

\subsubsection{NIR spectrometry}

\subsection{Calibration strategy}

To assess the ability of non-invasive NIR spectrometry to give accurate and precise prediction of alcohol content, the samples were split into calibration and test sets. As only a limited number of samples were available in this study, a variation of an algorithm based on a combination of principal component analysis (PCA) and PLS [21] (Figure 1) was used to select the minimum number of samples for the calibration model. Initially, data from three samples were designated as the calibration set and the remaining samples were assessed using the method shown in Figure 1. If a sample was deemed to be statistically different from those in the calibration set, the repeat 
spectra and alcohol concentration for the sample (provided by the manufacturer) were added to the calibration set. In comparison, if a sample was deemed to be statistically similar to those in the calibration set, the data were added to the test set. By using this process, the samples available were split into calibration and test sets.

An advantage of the original version of the PCA-PLS algorithm (Figure 2), as described in reference 21 , is that it can be applied on-line and in real-time where the future boundaries of the sample set may change as new unknown samples are considered. If a new sample is shown to be different from those in the calibration model, it can be identified for off-line analysis by a reference method, and the data used to update the PLS calibration model. This approach was simulated in the study.

The key difference between the two versions of the algorithm is that in Figure 1 a PLS model is constructed and applied only after all of the samples have been assessed while in Figure 2 the PLS model is updated and applied as new samples are considered.

\subsection{Method}

PCA is used to assess the similarity of new samples using the lack of fit, Q, statistic. This is achieved by comparison of the $\mathrm{Q}$ value for a new sample $\left(\mathrm{Q}_{\text {new }}\right)$, on projection into the sub-space defined by the PCA model for the current calibration samples, with the critical $Q$ value at a specified confidence limit $\left(\mathrm{Q}_{\text {crit }}\right)$, for the PCA model. The use of the Q statistic was preferred to Hotelling's $\mathrm{T}^{2}$ as it has been shown that $\mathrm{Q}$ is more likely to be normally distributed than $\mathrm{T}^{2}$ (calculation of the confidence limits assumes normal distribution of the data) [22]. If the $\mathrm{Q}$ value for the new sample is greater than that for the current PCA model, the sample is deemed to be dissimilar 
(i.e. contains additional information to that currently in the calibration set) and is added to the calibration sample set. In the on-line version of the algorithm (Figure 2), an updated PLS calibration model is then constructed to include the additional information. In comparison, if the Q value for the new sample is smaller than that for the current PCA model, the sample is similar to those in the existing calibration set and thus, the composition of the sample can be predicted.

The algorithm requires input of several choices: the number of initial calibration samples (n); the \% variance in the data to be retained by the PCA model (which defines the number of principal components (PCs) to be used); the number of PCs to be used for construction of the PLS models; the statistic to be used to assess sample similarity; the confidence limit for the statistic.

Data from five repeat spectra of samples 1-23, which were acquired on the same day using spectrometer 1, were assessed using the two PCA-PLS algorithms depicted in Figure 1 and Figure 2. First derivative spectra were mean centred and only the $7169-10437 \mathrm{~cm}^{-1}$ region was analysed. In order to select the initial calibration samples for the PCA-PLS procedures, the spectra were subjected to PCA. Three samples at the extremes of the region encompassed by the whisky and vodka samples within the subspace defined by a 2 PC PCA model (which captured $96 \%$ of the variance in the data) were selected (samples 4, 7 and 22). The whisky and vodka samples were considered first to ensure that the calibration set could model alcohol content well for the majority of samples available. The small number of sugary drink samples, which spanned a limited range in terms of alcohol and sugar content, were then assessed. The Q statistic was used to assess the similarity of the remaining 20 samples to the 3 samples initially selected. The \% variance to be captured by the PCA model was varied between 95 and $99 \%$, and the confidence limit for Q was varied between 80 
and $99 \%$, in order to determine the optimum values. The values selected (97\%) were those that gave the minimum standard error of prediction (SEP) with the smallest number of samples. When the PCA-PLS algorithm was employed to split the samples into calibration and test sets (Figure 1), five latent variables were used for the PLS model (contained all three types of samples). The number of latent variables required was determined from the model that gave the minimum value of the root mean square error of cross validation (RMSECV) obtained using leave-one-out cross validation. With the on-line version of the algorithm (Figure 2), three latent variables were employed for PLS models that contained only whisky and vodka samples, whereas five latent variables were required for PLS models which contained all three types of samples (as above).

\subsection{Predictive capability over time}

In order to study the predictive capability of a calibration model with time, samples 27-32 were analysed on 11 different days over a 15 day period using spectrometer 2. Five repeat measurements were made for the six samples on each day. A PLS calibration model was constructed on each of the 11 days from the spectra acquired of samples 28-32. The concentration of the remaining sample, sample 27, was then predicted. In addition, a PLS calibration model was constructed using only the data acquired of samples $28-32$ on the first day. This model was used to estimate the concentration of sample 27 based on the spectra of the sample acquired over the 15 day period. No pre-processing other than derivatisation was applied to the data, and only the $7169-10437 \mathrm{~cm}^{-1}$ region was analysed. Four latent variables were required for the PLS models. 


\subsubsection{Raman spectrometry}

Univariate calibration models were constructed using the signal at $873 \mathrm{~cm}^{-1}$ in the first derivative spectrum. Separate calibration models were constructed for each type of drink. The alcohol content of each of the samples, other than those with the highest (samples 14, 22 and 25) and lowest (samples 9, 16 and 26) alcohol content, were predicted using a calibration model constructed from all of the remaining samples of the same type so that the test sample was not included in the calibration set. Samples 12 and 13 exhibited fluorescence and were omitted from the data set for the sugary drinks. Hence, spectra from six, five and four samples were used to construct calibration models and thus predict the concentration of five whiskies, four vodkas and three sugary drinks, respectively.

\section{Results}

\subsection{Spectral interpretation}

\subsubsection{NIR spectrometry}

Examples of NIR spectra obtained for whisky, vodka and sugary drink samples, water and ethanol contained in $200 \mathrm{~mL}$ clear glass flat bottles are shown in Figure 3. Measurements were made across the narrowest part of the bottle. Most of the absorbance signals below $9000 \mathrm{~cm}^{-1}$ are off-scale due to the large pathlength $(35 \mathrm{~mm}$ $x$ 2) of the double transmission measurement made through the bottle. Therefore, in non-invasive analysis it is not possible to use the same spectral region as that 
employed in the off-line NIR method applied by Guinness United Distillers and Vintners. Spectra obtained for the different types of samples show the same features, although the regions $7500-8500 \mathrm{~cm}^{-1}$ and $9000-10500 \mathrm{~cm}^{-1}$ show the most response to changes in alcohol concentration. In the region $7000-9000 \mathrm{~cm}^{-1}$, the peak (off-scale) at $\sim 7000 \mathrm{~cm}^{-1}$ is from the $1^{\text {st }}$ overtone $\mathrm{O}-\mathrm{H}$ stretch (water and ethanol) and the peaks (off-scale) at $\sim 8500 \mathrm{~cm}^{-1}$ arise from the $2^{\text {nd }}$ overtone $\mathrm{C}-\mathrm{H}$ stretch (ethanol) and a combination of the $\mathrm{O}-\mathrm{H}$ bend and the $1^{\text {st }}$ overtone of the $\mathrm{O}-\mathrm{H}$ stretch (water) [4]. In the region $9000-10500 \mathrm{~cm}^{-1}$, the signal at $\sim 10000 \mathrm{~cm}^{-1}$ arises predominantly from the $2^{\text {nd }}$ overtone $\mathrm{O}-\mathrm{H}$ stretches of water $\left(\sim 10300 \mathrm{~cm}^{-1}\right)$ and ethanol $\left(\sim 9800 \mathrm{~cm}^{-1}\right)[4]$ and hence, exhibits a shift to lower wavenumbers as the alcohol concentration increases. However, changes in hydrogen bonding in the mixture compared to that in the pure components also affect the peak position. As the water signal is much stronger than that of ethanol, the composite $\mathrm{O}-\mathrm{H}$ peak decreases in intensity as the alcohol concentration increases. Plot d in Figure 3 shows a spectrum of one empty glass bottle when another empty bottle is used as the reference. If the two bottles were identical then the absorbance would be 0 at all wavenumbers. This was not the case, resulting in a baseline offset, which was removed through application of a first derivative to the data from all samples.

First derivative spectra of solutions of 20,30 and $40 \% \mathrm{~V} / \mathrm{V}$ ethanol were compared with those of a whisky (sample 6), vodka (sample 8) and sugary drink (sample 11), all with an ethanol concentration of approximately $20 \% \mathrm{~V} / \mathrm{V}$. Figure 4 shows that the spectra (in the $9500-10000 \mathrm{~cm}^{-1}$ region) of the whisky and vodka are in reasonable agreement with that of a $20 \% \mathrm{~V} / \mathrm{V}$ ethanol solution. However, the spectrum of the sugary drink is closer to that of the $30 \% \mathrm{~V} / \mathrm{V}$ ethanol solution. Although the $2^{\text {nd }}$ overtone $\mathrm{O}-\mathrm{H}$ stretch from sugars contributes to this spectral region 
[23], the observed change arises from the lower water content of a sugary drink compared to, e.g. a vodka sample of comparable ethanol concentration. In addition, sugars contribute directly to some spectral regions that also contain signals from ethanol, e.g., the $2^{\text {nd }}$ overtone C-H stretch region $\left(\sim 8300 \mathrm{~cm}^{-1}\right.$ in Figure 3$)$ [23]. This suggests that if a calibration model was constructed only from whisky, vodka or solutions of ethanol, the prediction of the alcohol content of sugary drinks would be positively biased.

\subsubsection{Raman spectrometry}

Examples of Raman spectra obtained for each of the three types of samples contained in $200 \mathrm{~mL}$ clear glass bottles are shown in Figure 5. For the spectra shown, the measurement was made across the widest part of bottle. It can be seen that the glass bottle makes a significant contribution to the overall spectrum (Figure 5, plot d). The glass spectrum was not subtracted from each sample spectrum, owing to differences in the bottles. The most significant feature in the spectra of the whisky and vodka (plot a and b, respectively, in Figure 5) is the signal at $880 \mathrm{~cm}^{-1}$, which can be attributed to the symmetric C-C-O stretch of ethanol. The main difference between the spectra of the whiskies and vodkas is the large fluorescent background caused by components in the whisky. Fluorescence from the sugary drink (Figure 5, plot c) was much greater and it was not possible to use Raman spectrometry to analyse some samples of this type of beverage. A major potential advantage of the Raman scattering measurements over the NIR method used in this work, is that the Raman spectra are caused by the ethanol content of the samples, allowing direct analysis, whereas the 
non-invasive NIR signals are dominated by water and thus require multivariate methods to derive the ethanol concentration.

The first derivative Raman spectra of a vodka (sample 8) and a sugary drink (sample 10) of equivalent alcohol content $(\sim 20 \% \mathrm{~V} / \mathrm{V})$ are shown in Figure 6. Although there are signals from additional components in the sugary drink, they do not overlap with the ethanol signal at $880 \mathrm{~cm}^{-1}\left(873 \mathrm{~cm}^{-1}\right.$ in the first derivative spectrum), which is identical for both samples.

\subsection{Assessment of factors affecting non-invasive measurements}

\subsubsection{NIR spectrometry}

The effects of glass colour and bottle diameter on spectra were assessed by analysing a solution of $28.8 \% \mathrm{~V} / \mathrm{V}$ ethanol in $200 \mathrm{~mL}$ and $700 \mathrm{~mL}$ clear glass bottles and in a $700 \mathrm{~mL}$ green glass bottle. The spectra obtained are shown in Figure 7. It can be seen in plot $b$ of Figure 7 that it is not possible to obtain a signal for the sample in a $700 \mathrm{~mL}$ bottle when the measurement is made across the widest part of the bottle (diameter of $70 \mathrm{~mm}$ ). However, if the measurement is made across the neck of the bottle (Figure 7, plot c), where the diameter is $40 \mathrm{~mm}$, the signal is comparable to that obtained for the $200 \mathrm{~mL}$ clear glass flat bottle (Figure 7, plot a). This highlights one of the potential problems of any transmission measurement in that the bottle diameter may limit its applicability. It can also be seen from plot $d$ in Figure 7 that the colour of the glass is important as in this case it was not possible to obtain a signal through a green glass bottle. 
As the techniques are being applied non-invasively, any differences in the position of the bottle and also between the bottles will affect the repeatability of the data. A solution of $20 \% \mathrm{~V} / \mathrm{V}$ ethanol was put into five different $200 \mathrm{~mL}$ clear glass flat bottles, and measurements were made across the narrowest (width of $35 \mathrm{~mm}$ ) part of the bottle to allow calculation of the short- and long-term instrumental measurement variance, the positional variance and the bottle-to-bottle variance. The average of the signal intensity at $9796 \mathrm{~cm}^{-1}$ was also calculated for the four sets of data. A summary of the data obtained is given in Table 1. It can be seen that the main contribution to the variability in the data was from differences between the bottles (RSD of $2.3 \%)$.

\subsubsection{Raman spectrometry}

The effects of glass colour and bottle diameter on Raman spectra were assessed by analysing a solution of $28.8 \% \mathrm{~V} / \mathrm{V}$ ethanol in $200 \mathrm{~mL}$ and $700 \mathrm{~mL}$ clear glass bottles and in a $700 \mathrm{~mL}$ green glass bottle. The spectra obtained are shown in Figure 8. It can be seen that the glass signal, obtained when the sample is analysed across the widest part of the $200 \mathrm{~mL}$ bottle (Figure 8 , plot a), is much less than when analysed across the narrowest part of the $200 \mathrm{~mL}$ bottle (Figure 8 , plot b). Unlike the NIR double transmission method, it is possible to obtain a signal across the widest part of the $700 \mathrm{~mL}$ clear glass bottle (Figure 8, plot c). This is one of the major advantages of Raman over transmission-based methods, as the signal generated is not limited by the bottle diameter. However, as for NIR spectrometry, the colour of the glass is important and in this case, the green bottle fluoresces too much for sensitive measurement of the ethanol spectrum (Figure 8, plot d). 
The contributions of measurement repeatability, bottle position and bottle-tobottle variability to the overall analytical variance were determined using a $20 \% \mathrm{~V} / \mathrm{V}$ ethanol solution contained in 200 and $700 \mathrm{~mL}$ clear glass bottles. In the former case, the sample was analysed across both the widest and narrowest parts of the bottles. The average intensity and variance of the signal at $873 \mathrm{~cm}^{-1}$ in the first derivative spectrum were calculated and used to give a measure of the magnitude of the instrumental, positional and bottle variability, expressed as the RSD. A summary of the variance data obtained is given in Table 2. The Raman measurement variance was calculated for the same period of time (equivalent to 25 repeat measurements) as for the positional and bottle variability measurements to account for laser power fluctuations. It can be seen that the bottle-to-bottle variance was larger when measurements were made across the widest part of the $200 \mathrm{~mL}$ bottle than across the narrowest part of the bottle. However, the lowest bottle variability was found for the round bottles. This is not surprising as the manufacturer's tolerances for these bottles are tighter than for the $200 \mathrm{~mL}$ flat glass bottles. The positional variation is also smaller for the round bottles.

\subsection{Determination of alcohol content}

\subsubsection{NIR spectrometry}

Twenty-three samples were analysed in the $200 \mathrm{~mL}$ clear glass bottles (across the narrowest part) and PCA was carried out on the $7169-10437 \mathrm{~cm}^{-1}$ region of the first derivative spectra. Five repeat spectra were obtained for each sample. The scores for PC 1 and 2 are shown in Figure 9. PC1, which described $91.6 \%$ of the variation in the data, is associated with ethanol (correlated) and water (anti-correlated), with the 
samples containing the highest alcohol content having the largest PC1 score. PC2, which described $4.4 \%$ of the variation in the data, is associated with peak shifts that arise from changes in ethanol-water composition and hydrogen bonding. It can be seen that there was no differentiation between sample type for whiskies and vodkas. However, the sugary drinks, which all have an alcohol content of approximately $20 \%$ $\mathrm{V} / \mathrm{V}$, had very different PC2 scores to the whiskies and vodkas, and the value of the PC1 score value was higher than expected for three of the four samples. This was not unexpected given the differences between the spectra of whisky and vodka samples and sugary drinks of comparable concentration (Figure 4).

The samples selected to add to the original calibration set $(4,7$ and 22$)$ were 1,8 and 10 as indicated in Figure 9. While samples 1 and 8 provide midpoints for the vertices of the calibration space for whiskies and vodkas (samples 4, 7 and 22), sample 10 extends the calibration space to encompass sugary drinks. The results for the test samples are given in Table 3. It was possible to predict the concentration of the whiskies and vodkas with an average $\%$ error of $1.6 \%$, where $\%$ error is defined as the difference between the predicted and actual concentration expressed as a percentage of the actual concentration. Prediction of the alcohol content of the three sugary drinks was less accurate $(4.5 \%)$, which was not unexpected given the dissimilarity of the samples in the PCA scores plot (Figure 9). Predictions for sugary drinks may be improved if a greater number of samples of this type, spanning a wider range in terms of alcohol and sugar content, could have been assessed and perhaps added to the PLS model. The precision (average RSD) with which the concentration can be predicted for all types of drinks was $0.4 \%$.

On-line application of the PCA-PLS algorithm (Figure 2) was simulated using the same data set. The sample spectra were compared at random to the initial PLS model 
built using samples 4, 7 and 22. The spectra of sample 1 were compared first and the content was found to be different to that in the model and so it was included to produce a revised PLS model. The updated model was then used to assess samples 3 and 14 , and both were found to be similar in information content to the model so their concentrations were predicted. Sample 8 was then assessed, found to be different and included in a revised model. The procedure was continued until the information content of the spectra of the remaining samples was assessed; the only other sample included in the PLS model was sample 10. Concentrations obtained for samples not in the calibration model did not vary significantly from the values given in Table 3 (average $\%$ error of $1.6 \%$ compared to $2.1 \%$ for all types of drinks).

The ability of a calibration model constructed on day 1 from five repeat measurements of five samples (28-32) to predict the concentration of sample 27 over 15 days can be seen in Table 4. On projection of the spectra obtained for sample 27 over 15 days into the sub-space defined by a PCA model (describing $>97 \%$ of the variance in the data) for samples 28-32 obtained on day 1, the Q value for each spectrum was less than the critical Q value at the $97 \%$ confidence limit for the PCA model. For comparison, the results obtained from prediction of the alcohol content of sample 27 using a calibration model constructed from data acquired on the same day are shown. In general, the predicted concentrations exhibit a negative bias although the concentrations predicted using the calibration models constructed on the same day as the test sample are approximately twice as accurate as those predicted using the model constructed on day 1 (average $\%$ error of 1.8 and $3.9 \%$, respectively). In addition, the predicted concentration over the 15 days is less precise using the model constructed on day 1 (average RSD of the 11 concentrations was $2.7 \%$ compared to $1.5 \%$ ). It should be possible to improve the predictive abilities of the model 
constructed on day 1 over time using instrument drift correction methods [24,25]. However, it would be expected that the variation in the predicted concentration of a particular product line from bottle-to-bottle variability would be greater than that observed from instrument drift (see Table 1). Results for day 9 are lower possibly owing to a large change in ambient temperature on this day compared to the rest; in the absence of temperature correction procedures, variations in temperature have a detrimental effect on the predictive ability of a PLS calibration model [7].

\subsubsection{Raman spectrometry}

Whiskies, vodkas and sugary drinks were analysed by non-invasive Raman spectrometry in the $700 \mathrm{~mL}$ clear glass round bottles, as these bottles had the lowest bottle-to-bottle variability. Univariate analysis of the signal intensity at $873 \mathrm{~cm}^{-1}$ in the first derivative was carried out, as there was a linear relationship between this signal and alcohol content. The slope \pm standard error for whiskies, vodkas and sugary drinks was $74.45 \pm 1.21,76.63 \pm 1.17$ and $73.06 \pm 1.30$ arbitrary units (a.u.) $/(\% \mathrm{~V} / \mathrm{V}$ alcohol $)$, respectively. Statistical analysis of the data using a t-test (assuming equal variances) suggested that the three slopes are different at the $95 \%$ confidence level. Although the signal at $873 \mathrm{~cm}^{-1}$ arises only from the alcohol, the sensitivity of response differs for the three types of samples possibly owing to differences in absorption and/or scattering of the laser or Raman scattered light [26]. Hence, three separate calibration models were required for the different types of alcoholic beverages. Due to the limited number of samples for each type of drink, it was not possible to divide the samples up into calibration and test sets. The concentration of each of the samples, other than those with the highest and lowest 
alcohol content, was predicted using a calibration model constructed from all of the remaining samples of that type. The predicted concentrations and the samples used to construct the calibration models are given in Table 5. It was possible to predict the alcohol concentration with an accuracy of 2.0, 3.5 and $3.6 \%$ average error and a precision of $0.5,0.2$ and $0.7 \%$ average RSD, for whisky, vodka and sugary drink samples, respectively.

\subsubsection{Comparison of Raman and NIR spectrometries}

The results obtained using NIR (Table 3) and Raman spectrometries (Table 5) for samples contained in clear glass 200 and $700 \mathrm{~mL}$ bottles, respectively, were compared. The effect of differences in calibration model size for the two techniques should be minimal as 6 samples were employed for NIR spectrometry and 6, 5 and 4 samples were employed for the whisky, vodka and sugary drink calibration models, respectively, for Raman spectrometry. For all types of samples with ethanol concentrations in the range $19.9-61.7 \% \mathrm{~V} / \mathrm{V}$, the precision of the two methods was found to be comparable (average RSD of 0.4 and $0.5 \%$ for NIR and Raman spectrometries, respectively). The accuracy of NIR spectrometry was slightly better than that for Raman spectrometry (average \% errors of 2.1 and $2.9 \%$, respectively). However, Raman spectrometry gives a direct measurement of alcohol content, unlike the NIR method, which is based mainly on the measurement of a composite water and ethanol signal, dominated by water, at $\sim 10000 \mathrm{~cm}^{-1}$. The Raman calibration models would be easier to update and maintain as they are univariate. However, an algorithm such as PCA-PLS could be used to automate the maintenance and update of a multivariate calibration model required for NIR spectrometry. If the model was to be 
applied over many days, then an instrument drift correction algorithm should be applied to the data prior to use of the PCA-PLS algorithm. The applicability of double transmission NIR spectrometry may also be limited by bottle diameter.

The accuracy of the non-invasive methods is not as good as that of an off-line NIR spectrometry procedure, which is typically $\pm 0.05 \% \mathrm{~V} / \mathrm{V}$ ethanol (i.e. $0.13 \%$ relative error at $40 \% \mathrm{~V} / \mathrm{V}$ ethanol). However, a far greater number of samples (up to 69) were used to build the off-line calibration model than were available for this study. It may be possible to tolerate the poorer accuracy of the on-line method if it were possible to analyse a greater number of samples in a production line than that currently permitted by the off-line NIR method. The off-line NIR method currently employed allows analysis of approximately 1 in every 10000 samples. The measurement time per sample for NIR and Raman spectrometries was $300 \mathrm{~ms}$ and 3 minutes, respectively, with the instruments used in this study. Therefore, at a bottling line speed of 300 bottles per minute, an average alcohol concentration could be obtained from the measurement of 1.5 and 900 bottles, respectively, for NIR and Raman spectrometries. Hence, use of either technique on-line would permit analysis of a significantly larger number of samples than the off-line NIR method employed currently.

\section{Conclusions}

The application of non-invasive NIR spectrometry for determination of alcohol content may be limited by the bottle diameter. Use of a transmission instrument, rather than the reflectance system used here, could allow measurements to be made through the widest part of $700 \mathrm{~mL}$ bottles. If it was possible to analyse samples 
through the neck of bottles using transmission measurements, then it may allow signals arising from combination and overtone $\mathrm{CH}$ stretching vibrations of ethanol and sugars at lower wavenumbers to be utilised. Such signals would thus allow direct determination of ethanol content in the presence of sugars.

Although Raman spectrometry has some advantages over NIR spectrometry particularly with regard to calibration, there may be some safety concerns about deployment of a laser-based technology in a process environment. For example, the area directly opposite the laser would have to be screened and procedures put in place for automatic shut-off of the laser should the fibre optic cable become damaged. In addition, the analysis area would have to be screened to avoid signals that might arise from background lighting, e.g., fluorescent room lights.

The present study concerned the study of static bottles, but in an active bottling hall the bottles would be moving. Given the analysis time of the techniques compared to the speed of the bottling line, the reported alcohol concentration would be an average over a number of bottles. The space between adjacent bottles on a bottling line would be minimal compared to the cross-section of the bottle seen by the analyser. Although, the glass and thus sample to analyser source distance would vary as the bottle passes by the analyser, owing to the curvature of the bottle, such effects would be averaged. It would also be important that bottles are presented consistently to the analyser to avoid labelling or joins on the bottle and to reduce variability owing to bottle positioning.

Non-invasive measurements could also be used for the non-destructive analysis of samples in bottles in a quality control laboratory. Although fewer samples would be analysed in comparison to an on-line application, samples could be analysed without being opened and thus could still be sold. Another potential application of 
non-invasive measurements of alcoholic beverages in bottles is in the area of counterfeit detection. Again this would allow samples to be tested without opening the bottles, and thus if the product was found to be legitimate, it could still be used.

\section{Acknowledgements}

The DTI and EPSRC are thanked for the award of a LINK grant (KNOW-HOW) under which AN and RTB were appointed (EPSRC grant no. GR/R19366/01). Guinness United Distillers and Vintners is thanked for the provision of samples and bottles. Clairet Scientific is thanked for the loan of one of the Zeiss Corona NIR instruments used in this work. 


\section{Figure captions}

Figure 1. PCA-PLS algorithm used to divide a pool of samples into calibration and test sets.

Figure 2. PCA-PLS algorithm used for maintenance and update of a calibration model [21].

Figure 3. NIR spectra of a) whisky $(56.7 \% \mathrm{~V} / \mathrm{V})$, b) vodka $(37.7 \% \mathrm{~V} / \mathrm{V})$, c) a sugary drink $(20.0 \% \mathrm{~V} / \mathrm{V}), \mathrm{d})$ an empty glass bottle, e) water and f) ethanol.

Figure 4. First derivative NIR spectra of solutions of 20, 30 and $40 \% \mathrm{~V} / \mathrm{V}$ ethanol (dashed lines), whisky, W (20.2 \% V/V), vodka, V (19.9\% V/V) and a sugary drink, $\mathrm{S}(20.1 \% \mathrm{~V} / \mathrm{V})$.

Figure 5. Raman spectra of a) whisky $(56.7 \% \mathrm{~V} / \mathrm{V}), \mathrm{b})$ vodka $(37.7 \% \mathrm{~V} / \mathrm{V})$, c) a sugary drink $(20.0 \% \mathrm{~V} / \mathrm{V})$ and d) an empty glass bottle.

Figure 6. First derivative Raman spectra of a) a vodka (19.9\% V/V) and b) a sugary $\operatorname{drink}(20.4 \% \mathrm{~V} / \mathrm{V})$

Figure 7. NIR spectra of a $28.8 \% \mathrm{~V} / \mathrm{V}$ ethanol solution in a) $200 \mathrm{~mL}$ clear glass bottle (measured across narrowest part), b) $700 \mathrm{~mL}$ clear glass bottle (measured across widest part), c) $700 \mathrm{~mL}$ clear glass bottle (measured across neck) and d) $700 \mathrm{~mL}$ green glass bottle (measured across neck).

Figure 8 . Raman spectra of a $28.8 \% \mathrm{~V} / \mathrm{V}$ ethanol solution in a) $200 \mathrm{~mL}$ clear glass bottle (measured across narrowest part), b) $200 \mathrm{~mL}$ clear glass bottle (measured 
across widest part), c) $700 \mathrm{~mL}$ clear glass bottle (measured across widest part) and d) $700 \mathrm{~mL}$ green glass bottle (measured across widest part).

Figure 9. PC1 v PC2 scores plot from PCA of NIR spectra of samples 1-23. Samples that were chosen initially $(4,7$ and 22$)$ to form the calibration set and those that were selected in addition (1, 8 and 10) are indicated. 


\section{Table captions}

Table 1. Variance in NIR signals associated with the measurement (short- and long-term), positional and bottle variability for a $20 \% \mathrm{~V} / \mathrm{V}$ ethanol solution in $200 \mathrm{~mL}$ clear glass bottles.

Table 2. Variance in the $1^{\text {st }}$ derivative Raman signal at $873 \mathrm{~cm}^{-1}$ associated with the measurement, positional and bottle variability for a $20 \% \mathrm{~V} / \mathrm{V}$ ethanol solution in $200 \mathrm{~mL}$ (measured across widest and narrowest parts) and $700 \mathrm{~mL}$ clear glass bottles (measured across widest part).

Table 3. Predicted alcohol concentrations obtained using PLS analysis of NIR spectra. $^{\#}$

Table 4. Prediction of the alcohol content of sample 27 (whisky; $33.1 \%$ V/V) over a 15 day period using a calibration model constructed on day 1 and calibration models constructed from data acquired on the same day. ${ }^{\#}$

Table 5. Predicted alcohol concentrations obtained using univariate analysis of Raman spectra. 


\section{References}

[1] M. Gallignani, S. Garrigues and M. de la Guardia, Anal. Chim Acta, 287 (1994) 275.

[2] M. Gallignani, S. Garrigues and M. de la Guardia, Analyst, 119 (1994) 1773.

[3] S.A. Halsey, Anal. Proc., 23 (1986) 126.

[4] S. Engelhard, H.-G. Löhmannsröben and F. Schael, Appl. Spectrosc., 58 (2004) 1205.

[5] M. Gallignani, S. Garrigues and M. de la Guardia, Analyst, 118 (1993) 1167.

[6] L.S. Mendes, F.C.C. Oliveira, P.A.Z. Suarez and J.C. Rubin, Anal. Chim. Acta, $493(2003) 219$.

[7] F.D. Barboza and R.J. Poppi, Anal. Bioanal. Chem., 377 (2003) 695.

[8] J. Sjoblom, O. Svensson, M. Josefson, H. Kullberg and S. Wold, Chemom. Intell. Lab. Syst., 44 (1998) 229.

[9] Y. Wang, D.J. Veltkamp and B.R. Kowalski, Anal. Chem., 63 (1991) 2750.

[10] C.L. Sanford, B.A. Mantooth and B.T. Jones, J. Chem. Educ., 78 (2001) 1221.

[11] R.Y. Sato-Berrú, J. Medina-Valtierra, C. Medina-Gutiérrez and C. FraustoReyes, Spectrochim. Acta A, 60 (2004) 2225.

[12] M. Gallignani, S. Garrigues and M. de la Guardia, Anal. Chim Acta, 296 (1994) 155.

[13] C.-D. Patz, A. Blieke, R. Ristow and H. Dietrich, Anal. Chim Acta, 513 (2004) 81.

[14] A. Urtubia, J.R. Pérez-Correa, M. Meurens and E. Agosin, Talanta, 64 (2004) 778.

[15] W.M. MacKenzie and R.I. Aylott, Analyst, 129 (2004) 607. 
[16] B.R. Buchanan, D.E. Honigs, C.J. Lee and W. Roth, Appl. Spectrosc., 42 (1988) 1106.

[17] F.W.J. van den Berg, W.A. van Osenbruggen and A.K. Smilde, Proc. Control Qual., 9 (1997) 51.

[18] R.J. Ampiah-Bonney and A.D. Walmsley, Analyst, 124 (1999) 1817.

[19] A.G. Cavinato, D.M. Mayes, Z. Ge and J.B. Callis, Anal. Chem., 62 (1990) 1977.

[20] N.W. Broad, R.D. Jee, A.C. Moffat, M.J. Eaves, W.C. Mann and W. Dziki, Analyst, 125 (2000) 2054.

[21] S.K. Setarehdan, J.J. Soraghan, D. Littlejohn and D.A. Sadler, Anal. Chim. Acta, $452(2002) 35$.

[22] B.M. Wise and N.B. Gallagher, PLS_Toolbox 2.1, Eigenvector Research Inc., Manson, WA, 2000, p. 34-36.

[23] M. Golic, K. Walsh and P. Lawson, Appl. Spectrosc., 57 (2003) 139.

[24] R.N. Feundale, N.A. Woody, H.W. Tan, A.J. Myles, S.D. Brown and J. Ferre, Chemom. Intell. Lab. Syst., 64 (2002) 181.

[25] C.M. Wehlburg, D.M. Haaland, D.K. Melgaard and L.E. Martin, Appl. Spectrosc., 56 (2002) 605.

[26] P.J. Aamoutse and J.A. Westerhuis, Anal. Chem., 77 (2005) 1228. 
Table 1. Variance in NIR signals associated with the measurement (short- and long-term), positional and bottle variability for a $20 \% \mathrm{~V} / \mathrm{V}$ ethanol solution in $200 \mathrm{~mL}$ clear glass bottles.

\begin{tabular}{|c|c|c|c|c|c|c|}
\hline \multirow{2}{*}{$\mathrm{n}$} & \multirow{2}{*}{$\begin{array}{c}1^{\text {st }} \text { derivative } \\
\text { average signal at } \\
9796 \mathrm{~cm}^{-1} \text { (a.u.) }\end{array}$} & \multicolumn{2}{|c|}{ Total variance } & \multicolumn{2}{|c|}{ Individual variance } & \multirow{2}{*}{$\begin{array}{l}\text { RSD } \\
(\%)\end{array}$} \\
\hline & & source $^{\dagger}$ & $10^{-9} \times(\text { a.u. })^{2}$ & source & $10^{-9} \times(\text { a.u. })^{2}$ & \\
\hline 5 & $-0.0543^{\dagger}$ & M & 3.7 & M & 3.7 & 0.1 \\
\hline 25 & $-0.0541^{\dagger}$ & $\mathrm{M}+\mathrm{P}$ & 43.5 & $\mathrm{P}$ & 39.8 & 0.4 \\
\hline 25 & $-0.0564^{t}$ & $\mathrm{M}+\mathrm{P}+\mathrm{B}$ & 1741.2 & B & 1693.9 & 2.3 \\
\hline 55 & $-0.0514^{*}$ & $\mathrm{M}+\mathrm{P}+\mathrm{T}$ & 186.7 & $\mathrm{~T}$ & 147.0 & 0.7 \\
\hline
\end{tabular}

${ }^{\dagger} \mathrm{M}=$ =short-term measurement, $\mathrm{P}=$ positional, $\mathrm{B}=$ bottle, $\mathrm{T}=$ long-term measurement.

$\$$ spectrometer 1 .

* spectrometer $2(\mathrm{M}=0.1 \% \mathrm{RSD})$. 
Table 2. Variance in the $1^{\text {st }}$ derivative Raman signal at $873 \mathrm{~cm}^{-1}$ associated with the measurement, positional and bottle variability for a $20 \% \mathrm{~V} / \mathrm{V}$ ethanol solution in $200 \mathrm{~mL}$ (measured across widest and narrowest parts) and $700 \mathrm{~mL}$ clear glass bottles (measured across widest part).

\begin{tabular}{|c|c|c|c|c|}
\hline \multirow{2}{*}{$\mathrm{n}$} & \multirow{2}{*}{ source $^{\dagger}$} & $\begin{array}{c}\mid 3 \\
\text { R00 mL round bottle } \\
\text { (across widest part) }\end{array}$ & $\begin{array}{c}200 \mathrm{~mL} \text { flat bottle } \\
\text { (across narrowest part) }\end{array}$ & $\begin{array}{r}200 \mathrm{~mL} \text { flat bottle } \\
\text { (across widest part) }\end{array}$ \\
\cline { 3 - 5 } 25 & $\mathrm{M}$ & 1.0 & 0.9 & 0.3 \\
\hline 25 & $\mathrm{P}$ & 0.3 & 1.5 & 0.9 \\
\hline 25 & $\mathrm{~B}$ & 0.9 & 1.4 & 6.3 \\
\hline 25 & Total & 1.4 & 2.2 & 6.4 \\
\hline
\end{tabular}

${ }^{\dagger} \mathrm{M}=$ measurement, $\mathrm{P}=$ positional, $\mathrm{B}=$ bottle. 
Table 3. Predicted alcohol concentrations obtained using PLS analysis of NIR spectra."

\begin{tabular}{|c|c|c|c|}
\hline Sample no. & Sample type & $\begin{array}{l}\text { Actual concentration/ } \\
\qquad(\% \mathrm{~V} / \mathrm{V})^{*}\end{array}$ & $\begin{array}{l}\text { Predicted concentration/ } \\
\qquad(\% \mathrm{~V} / \mathrm{V})^{\dagger}\end{array}$ \\
\hline 2 & $\mathrm{~W}$ & 47.5 & $46.7 \pm 0.2$ \\
\hline 3 & $\mathrm{~W}$ & 42.8 & $42.0 \pm 0.1$ \\
\hline 5 & $\mathrm{~W}$ & 33.3 & $31.1 \pm 0.1$ \\
\hline 6 & $\mathrm{~W}$ & 20.2 & $20.3 \pm 0.2$ \\
\hline 9 & $\mathrm{~V}$ & 19.9 & $20.0 \pm 0.1$ \\
\hline 11 & $\mathrm{~S}$ & 20.1 & $22.1 \pm 0.2$ \\
\hline 12 & $\mathrm{~S}$ & 20.0 & $20.3 \pm 0.2$ \\
\hline 13 & $\mathrm{~S}$ & 20.1 & $19.9 \pm 0.1$ \\
\hline 14 & $\mathrm{~W}$ & 61.7 & $61.7 \pm 0.1$ \\
\hline 15 & $\mathrm{~W}$ & 40.1 & $40.1 \pm 0.0$ \\
\hline 16 & $\mathrm{~W}$ & 26.9 & $26.3 \pm 0.1$ \\
\hline 17 & $\mathrm{~W}$ & 58.1 & $57.9 \pm 0.1$ \\
\hline 18 & $\mathrm{~W}$ & 41.2 & $41.2 \pm 0.1$ \\
\hline 19 & $\mathrm{~V}$ & 40.2 & $38.0 \pm 0.1$ \\
\hline 20 & $\mathrm{~V}$ & 37.7 & $38.0 \pm 0.1$ \\
\hline 21 & $\mathrm{~V}$ & 45.1 & $44.5 \pm 0.2$ \\
\hline 23 & $\mathrm{~V}$ & 22.2 & $22.4 \pm 0.1$ \\
\hline
\end{tabular}

\# samples used to build the PLS calibration model were: whisky samples 1 (56.7\% $\mathrm{V} / \mathrm{V}), 4(38.0 \% \mathrm{~V} / \mathrm{V})$ and $7(20.2 \% \mathrm{~V} / \mathrm{V})$; vodka samples $8(19.9 \% \mathrm{~V} / \mathrm{V})$ and 22 $(63.27 \% \mathrm{~V} / \mathrm{V})$; sugary drink sample $10(20.4 \% \mathrm{~V} / \mathrm{V})$.

* W=whisky, $\mathrm{V}=$ vodka, $\mathrm{S}=$ sugary drink.

* determined by off-line NIR spectrometry at Guinness United Distillers and Vintners (estimated average error of $\pm 0.05 \% \mathrm{~V} / \mathrm{V}$ ).

$\dagger$ average \pm standard deviation $(n=5)$. 
Table 4. Prediction of the alcohol content of sample 27 (whisky; $33.1 \%$ V/V) over a 15 day period using a calibration model constructed on day 1 and calibration models constructed from data acquired on the same day."

\begin{tabular}{|c|c|c|}
\hline \multirow{2}{*}{ Day } & \multicolumn{2}{|c|}{ Predicted concentration/(\% V/V) } \\
\cline { 2 - 3 } & $\begin{array}{c}\text { Calibration model } \\
\text { constructed on day } 1\end{array}$ & $\begin{array}{c}\text { New calibration model } \\
\text { constructed every day }\end{array}$ \\
\hline 1 & $33.0 \pm 0.1$ & $33.0 \pm 0.1$ \\
\hline 2 & $31.7 \pm 0.1$ & $32.7 \pm 0.1$ \\
\hline 3 & $31.8 \pm 0.0$ & $33.0 \pm 0.0$ \\
\hline 4 & $31.0 \pm 0.1$ & $32.1 \pm 0.1$ \\
\hline 5 & $31.1 \pm 0.1$ & $32.8 \pm 0.1$ \\
\hline 8 & $31.4 \pm 0.1$ & $32.5 \pm 0.1$ \\
\hline 9 & $30.7 \pm 0.0$ & $31.4 \pm 0.1$ \\
\hline 10 & $32.4 \pm 0.1$ & $33.0 \pm 0.1$ \\
\hline 11 & $32.0 \pm 0.1$ & $32.7 \pm 0.1$ \\
\hline 12 & $33.6 \pm 0.1$ & $32.5 \pm 0.1$ \\
\hline 15 & $32.5 \pm 0.1$ & $32.4 \pm 0.1$ \\
\hline
\end{tabular}

\# samples used to build the PLS calibration model were: whisky samples $28(61.2 \%$ $\mathrm{V} / \mathrm{V})$ and $29(41.2 \% \mathrm{~V} / \mathrm{V})$; vodka samples $30(37.6 \% \mathrm{~V} / \mathrm{V}), 31(63.0 \% \mathrm{~V} / \mathrm{V})$ and 32 $(21.7 \% \mathrm{~V} / \mathrm{V})$. 
Table 5. Predicted alcohol concentrations obtained using univariate analysis of Raman spectra.

\begin{tabular}{|c|c|c|c|c|}
\hline $\begin{array}{c}\text { Sample } \\
\text { no. }\end{array}$ & $\begin{array}{c}\text { Sample } \\
\text { type }\end{array}$ & $\begin{array}{c}\text { Actual concentration/ } \\
(\% \mathrm{~V} / \mathrm{V})^{*}\end{array}$ & $\begin{array}{c}\text { Predicted concentration/ } \\
(\% \mathrm{~V} / \mathrm{V})^{\dagger}\end{array}$ & $\begin{array}{c}\text { Calibration } \\
\text { samples }^{\#}\end{array}$ \\
\hline 1 & $\mathrm{~W}$ & 56.7 & $57.8 \pm 0.1$ & $2,3,4,14,16,18$ \\
\hline 2 & $\mathrm{~W}$ & 47.5 & $48.7 \pm 0.1$ & $1,3,4,14,16,18$ \\
\hline 3 & $\mathrm{~W}$ & 42.8 & $41.3 \pm 0.4$ & $1,2,4,14,16,18$ \\
\hline 4 & $\mathrm{~W}$ & 38.0 & $37.4 \pm 0.1$ & $1,2,3,14,16,18$ \\
\hline 18 & $\mathrm{~W}$ & 41.2 & $41.1 \pm 0.3$ & $1,2,3,4,14,16$ \\
\hline 21 & $\mathrm{~V}$ & 45.1 & $43.2 \pm 0.1$ & $8,9,20,22,23$ \\
\hline 20 & $\mathrm{~V}$ & 37.7 & $39.2 \pm 0.0$ & $8,9,21,22,23$ \\
\hline 23 & $\mathrm{~V}$ & 22.2 & $23.7 \pm 0.1$ & $8,9,20,21,22$ \\
\hline 8 & $\mathrm{~V}$ & 19.9 & $20.1 \pm 0.1$ & $9,20,21,22,23$ \\
\hline 24 & $\mathrm{~S}$ & 23.7 & $24.1 \pm 0.1$ & $10,11,25,26$ \\
\hline 10 & $\mathrm{~S}$ & 20.4 & $19.3 \pm 0.4$ & $11,24,25,26$ \\
\hline 11 & $\mathrm{~S}$ & 20.1 & $20.8 \pm 0.0$ & $10,24,25,26$ \\
\hline
\end{tabular}

$\$ \mathrm{~W}=$ whisky, $\mathrm{V}=$ vodka, $\mathrm{S}=$ sugary drink.

${ }^{\dagger}$ average \pm standard deviation $(\mathrm{n}=3)$

* determined by off-line NIR spectrometry at Guinness United Distillers and Vintners (estimated average error of $\pm 0.05 \% \mathrm{~V} / \mathrm{V}$ ).

\# Details for samples 1-23 are given in Table 3. Samples $25(35.6 \% \mathrm{~V} / \mathrm{V})$ and 26 $(11.4 \% \mathrm{~V} / \mathrm{V})$ are sugary drinks. 